\title{
Analysis of Optical Frequency Comb Generation in Gain-Switched Semiconductor Lasers
}

\author{
A. Rosado , A. Pérez-Serrano , J.M.G Tijero , A. Valle , L. Pesquera and I. Esquivias
}

An Optical Frequency Comb (OFC) Generator is a laser source emitting an equally spaced group of optical tones. These types of optical sources have found application in different fields, such as spectroscopy [1] and optical communications [2]. Among the three main techniques for generating OFCs from semiconductor lasers, namely gain switching, electro-optic modulation and mode-locking, gain switching (GS) has attracted attention due to the simple selection of the repetition frequency and its easy implementation and low cost.

In the past, some spectral features of OFCs generated by GS were analysed, either experimentally [3], or theoretically [4]. We present here an exhaustive and systematic comparative analysis of the OFC generation using GS in semiconductor lasers. We have used a theoretical model, based on three stochastic differential equations that include non-linear saturation and Langevin noises, in order to simulate the experimental results. The experimental and simulated response has been studied in order to identify the main physical processes involved in the generation of GS OFC. An experimental characterization, based on RIN spectra measurements [5], was used to extract the parameters of the model. The OFC generator consists on a Discrete Mode Laser (DML), which is driven in GS operation using a bias current and a sinusoidal signal. High-resolution spectra were measured with a Brillouin Optical Spectrum Analyzer (BOSA).
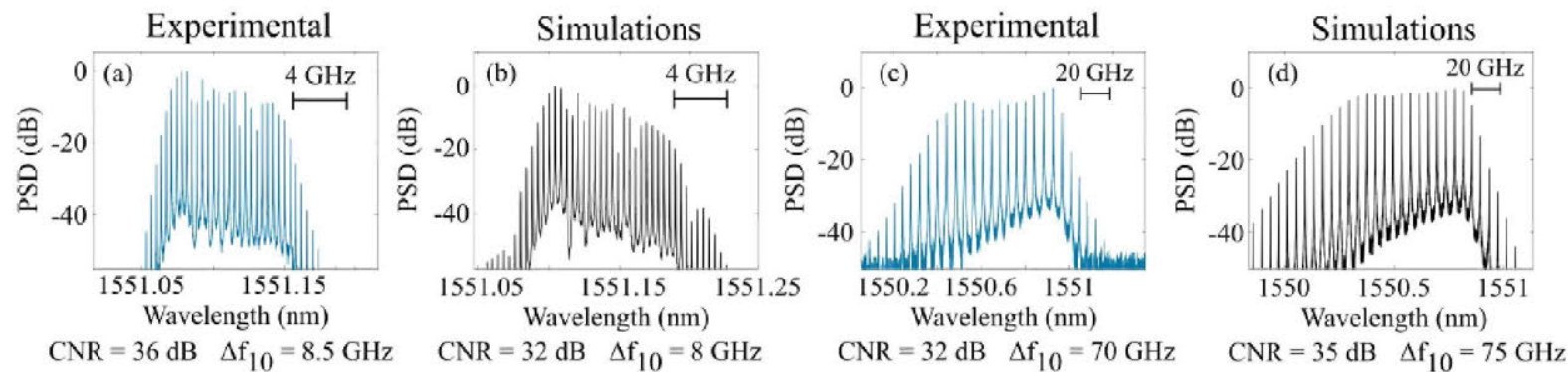

Fig. 1. Experimental and simulated optical spectra of the gain-switched DML laser in different driving conditions: (a) and

(b), $\mathrm{I}_{\text {bias }}=60 \mathrm{~mA}, \mathrm{~V}_{\mathrm{rf}}=1.5 \mathrm{~V}, \mathrm{f}_{\mathrm{R}}=500 \mathrm{MHz}$, and (c) and (d), $\mathrm{I}_{\text {bias }}=40 \mathrm{~mA}, \mathrm{~V}_{\mathrm{rf}}=1.5 \mathrm{~V}, \mathrm{f}_{\mathrm{R}}=5 \mathrm{GHz}$.

Fig. 1 shows the experimental and simulated optical spectra of the gain-switched DML under different driving conditions. It is apparent that the experimental and simulated spectra are not only qualitative but also quantitavely very similar. At a modulation frequency of $500 \mathrm{MHz}$, both optical spectra (Figs. 1(a) and (b)) exhibit similar characteristics in terms of shape, carrier-to-noise ratio (CNR) and spectral width within $10 \mathrm{~dB}\left(\Delta \mathrm{f}_{10}\right)$. Both spectra show narrow OFCs $\left(\Delta \mathrm{f}_{10} \approx 8 \mathrm{GHz}\right)$ with high values of CNR (36 and $32 \mathrm{~dB}$, respectively) and a clear asymmetry with some suppressed tones. The tone suppression is a typical feature of frequency modulation (FM). The spectral performance is slightly different for higher frequencies. At $5 \mathrm{GHz}$, Figs.1(c) and (d) show OFCs which CNR values are similar to those observed in Fig. 1 (a) and (b). However, in terms of flatness and $\Delta \mathrm{f}_{10}$, their characteristics are clearly superior. For this driving conditions $\left(f_{R}=5 \mathrm{GHz}\right)$, the modulation frequency is close to the frequency of the relaxation oscillations, so that, the FM efficiency is high and the generated OFCs are broader. We have observed that the similarity between experimental and simulated spectra remains for the full range of driving conditions. The comparison between the simulated and the experimental behaviour has provided a clear understanding of the main physical processes involved in OFC generation. In the first case $\left(f_{R}=500 \mathrm{MHz}\right)$, the carrier dynamics induces frequency chirp due to the dependence of the carrier density on the photon density, through the non-linear gain saturation (adiabatic chirp). However, for $\mathrm{f}_{\mathrm{R}}=5 \mathrm{GHz}$, the frequency chirp is induced by the carrier dynamics during the generation of the pulse (dynamic chirp). The analysis of the simulations has allowed us to understand the physical mechanisms involved in the generation of OFC, as well as to predict the behaviour of these devices beyond the experimental limitations.

[1] I. Coddington, N. Newbury, and W. Swann, "Dual-comb spectroscopy," Optica, vol. 3, p. 414, apr 2016.

[2] M. Imran, P. M. Anandarajah, A. Kaszubowska-Anandarajah, N. Sambo, and L. Poti, "A survey of optical carrier generation techniques for terabit capacity elastic optical networks". IEEE Commun. Surv. Tutor., 20(1), 211-263, 2018.

[3] A. Rosado, A. Pérez-Serrano, J. M. G. Tijero, A. Valle, L. Pesquera, and I. Esquivias, "Experimental study of optical frequency comb generation in gain-switched semiconductor lasers". Opt. Laser. Technol., 108, 542-550, 2018

[4] P. M. Anandarajah, S. P. O. Duill, Rui Zhou, and L. P. Barry, "Enhanced Optical Comb Generation by Gain-Switching a Single-Mode Semiconductor Laser Close to Its Relaxation Oscillation Frequency," IEEE J. Sel. Top. Quantum Electron, vol. 21, pp. 592-600, nov 2015. [5] I. Fatadin, D. Ives, and M. Wicks, "Numerical Simulation of Intensity and Phase Noise From Extracted Parameters for CW DFB Lasers," IEEE J. Quantum Electron, vol. 42, pp. 934-941, sep 2006. 